\title{
Numeraire Dependence in Risk-Neutral Probabilities of Event Outcomes
}

Hanke, Michael; Poulsen, Rolf; Weissensteiner, Alex

Published in:

Journal of Derivatives

DOI:

10.3905/jod.2019.26.4.128

Publication date:

2019

Document version

Peer reviewed version

Document license:

Unspecified

Citation for published version (APA):

Hanke, M., Poulsen, R., \& Weissensteiner, A. (2019). Numeraire Dependence in Risk-Neutral Probabilities of Event Outcomes. Journal of Derivatives, 26(4), 128-143. https://doi.org/10.3905/jod.2019.26.4.128 


\title{
Numeraire Dependence in Risk-Neutral Probabilities of Event Outcomes
}

\author{
Michael Hanke, Rolf Poulsen, and Alex Weissensteiner*
}

\begin{abstract}
For most non-financial events, risk-neutral outcome probabilities are identical across numeraire currencies. Some events, however, such as elections or referendums, may have an impact on exchange rates. This implies numeraire dependence in risk-neutral outcome probabilities, which leads to different state prices for affected currency pairs. If betting odds available to punters do not reflect these differences, this may give rise to (approximate) arbitrage opportunities. Despite the sizable risk this creates, odds quoted by bookmakers seem to ignore this effect.
\end{abstract}

JEL classification: G13, F31.

Keywords: betting odds, numeraire dependence, political events.

*Prof. Michael Hanke (corresponding author), Michael.Hanke@uni.li, University of Liechtenstein, Institute for Finance, Fuerst-Franz-Josef-Strasse, 9490 Vaduz, Liechtenstein, P: +4232651155; Prof. Rolf Poulsen, rolf@math.ku.dk, University of Copenhagen, Department of Mathematical Sciences, Universitetsparken 5, 2100 Copenhagen, Denmark, P: +4535320685; Prof. Alex Weissensteiner, Alex.Weissensteiner@unibz.it, Free University of Bozen-Bolzano, School of Economics and Management, Universitaetsplatz 1, 39100 Bozen, Italy, P: +390471013496 . We thank the editor and an anonymous referee for helpful comments and suggestions. 


\section{Introduction}

Risk-neutral probabilities of event outcomes can be inferred from betting odds, which are just the state prices for these outcomes expressed according to betting market conventions. Nowadays, betting services are offered online and across borders. During the registration process on the corresponding websites, punters have the choice of a preferred currency from a pool of currencies offered. The chosen currency is then used for all transactions on this website 1 Aside from the traditional bookmakers' business model taken online, a newer type of betting marketplace known as betting exchanges allows punters also to sell (in betting jargon: to "lay") the outcome. An inspection of major betting websites shows that whereas betting odds typically differ slightly among bookmakers and betting exchanges, the odds quoted on any particular website are identical across all available transaction currencies. As pointed out by Neuberger (2016), this may give rise to potentially very profitable strategies involving bets on different outcomes in different currencies if exchange rates react to the outcomes differently and predictably.

There is a sizable literature analyzing the efficiency of various betting markets, which also identifies a number of potential behavioral biases in betting odds (see, e.g., Williams and Siegel, 2013). One such behavioral reason for observing different odds in different countries/currencies is home bias, which may tilt perceived outcome probabilities depending on the country of residence and, hence, currencies. This bias may lead bookmakers to quote, e.g., different odds in Italy and Brazil for bets on the result of a soccer game between their national teams (see, e.g., Golec and Tamarkin, 1991). From a neoclassical perspective, different risk-free interest rates may lead to differences in betting odds across transaction currencies (even if exchange rates are independent of event outcomes). However, these differences are typically small and easily hidden/absorbed in the bookmaker's "cut", which is betting

\footnotetext{
${ }^{1}$ For an overview of transaction currencies offered by large online bookmakers, see https://tinyurl.com/y9clv6nh.
} 
jargon for the bid-ask spread implied in betting odds. In this paper, we analyze a neoclassical reason for currency dependence of betting odds, which seems to have received almost no attention so far from neither academia nor practice, and which lies at the heart of the strategies suggested by Neuberger (2016). It occurs for certain political events such as elections or referendums, which are expected to affect exchange rates. As two recent examples for such events, consider the U.K. Brexit referendum and the U.S. presidential elections. In June 2016, the British pound fell sharply after a majority in the U.K. voted in favor of leaving the European Union. This downward jump in the exchange rate reflected market expectations of negative economic effects resulting from this referendum outcome. In November 2016, the Mexican peso weakened markedly after Donald Trump was elected U.S. president. This resulted from remarks Trump had made during his election campaign regarding plans to impose high tariffs on many goods imported from Mexico to protect U.S. industry, and to build a wall along the U.S.-Mexican border.

Neuberger (2016) proposes a strategy combining bets in pounds and euros, which is similar to the strategies used in this paper to derive no-arbitrage conditions. He points out that the strategy is risky, because exchange rates might react differently than expected, and he is worried that "much, if not all, of your profit will be eaten up by the bookie's spread and any commission charges you have to pay". We will show that this was not the case for bets on the Brexit referendum, and that the only requirement to make a profit was to correctly forecast a weakening of the pound in case of a majority voting for Leave, regardless of its magnitude. Finally, Neuberger asks himself why bookmakers do not change the odds according to the currency of the bet. His explanation is that while internationally active bookmakers are used to having significant currency exposures across their books, most bets are unrelated to exchange rate movements. Given that political betting makes up only a relatively small share of their business, Neuberger concludes that "the amount they are likely to lose from people exploiting the current 
system is probably small". Relative to the ideas presented by Neuberger (2016) for a general audience and in everyday language, the contribution of the present paper can be seen in providing a formal financial economics basis for the strategies he suggests and a detailed analysis of the underlying effect itself, the impact of transaction costs, and the risk-adjusted return of the cross-currency betting strategies. Under the simplifying assumption of perfect ex ante information on conditional (i.e., outcome-dependent) exchange rate reactions after the respective events, we derive no-arbitrage conditions. Relaxing this assumption and acknowledging the risk associated with conditional exchange rate movements turns the arbitrage opportunities into good deals Cochrane and Saa-Requejo, 2002) or approximate arbitrage opportunities (i.e., strategies with a very favorable Sharpe ratio, see Shanken, 1992). Arbitrage opportunities in betting markets have been documented in the literature, see e.g. Dixon and Pope (2004) and Vlastakis, Dotsis, and Markellos (2009) for arbitrage opportunities in bookmaker betting, or Franck, Verbeek, and Nüesch (2013) for inter-market arbitrage using bets placed with bookmakers and betting exchanges. In contrast, Wolfers and Zitzewitz (2004) compare prices on two major betting exchanges and find that arbitrage opportunities are virtually absent. Our results in this paper show that even though the no-arbitrage conditions traditionally checked in this literature are satisfied, this does not protect bookmakers from being exploited via approximate arbitrage strategies with very favorable Sharpe ratios and low loss probabilities. This is followed by a numerical illustration using real-world data from the Brexit referendum and the U.S. presidential elections 2016.

Hanke, Poulsen, and Weissensteiner (2019) show how information from betting odds can be combined with risk-neutral densities estimated from FX option prices to arrive at conditional exchange rate forecasts around such events. They note (see their footnote 9) that they "have no indication of systematic differences across geographical regions although theoretically, the risk-neutral event probabilities might differ slightly depending on the nu- 
meraire currency." In the present paper, we analyze why and by how much risk-neutral outcome probabilities (and betting odds) for such events should differ across currencies. In contrast to other effects documented in the literature, the reason for this difference is not behavioral, but purely neoclassical. So far, the effect we describe in this paper does not seem to be reflected in bookmakers' quoted odds, as we could not find any hint to currencydependent quotes on any of the major betting websites. Given the size of these political betting markets, the resulting risk for bookmakers is not to be underestimated: The largest internet betting exchange, Betfair, alone reported a total betting volume of 127 million pounds for the Brexit referendum ${ }^{2}$ and around 200 million pounds for the U.S. presidential elections ${ }^{3}$

The relevance of our results extends beyond betting markets. The effects analyzed in this paper occur whenever event risk is correlated with exchange rates, and when this event risk is tradable via financial contracts in different currencies. One example for such a situation are Credit Default Swaps (CDS), where the probability of the credit event may depend on exchange rate movements. Lando and Nielsen (2018) analyze this effect and show that CDS spreads on a particular reference entity should be currency-dependent if exchange rate fluctuations and the default intensity of the reference entity are correlated. There are a number of differences between their model and our setting, however: Whereas the political events analyzed in this paper affect exchange rates, exchange rate movements affect the occurrence of credit events. Moreover, the event dates in Lando and Nielsen (2018) are uncertain, and interest rate uncertainty is an additional important driver for CDS spreads, which leads them to a different modeling approach.

The paper is organized as follows: In Section 2, we present our model and describe the effect that is the focus of this paper. Section 3 analyzes the consequences of the observed mispricing in betting markets. Section 4 provides

\footnotetext{
$\sqrt[2]{\text { https://tinyurl.com/lgjzo3a }}$

3 https://tinyurl.com/m4mrkpn
} 
a numerical illustration of these consequences, and Section 5 concludes.

\section{Model}

For simplicity of exposition, we explain our model based on the Brexit referendum. The derivation in the case of the U.S. presidential elections (not shown) works along the same lines. Both cases will be illustrated numerically in Section 4. We use the terminology of financial economics, relating it to betting jargon where appropriate. Our notation is based on that used in Hanke et al. (2019), making it more precise where needed in the present context.

\subsection{Risk-neutral Event Outcome Probabilities Implied in Betting Odds}

Let time $\tau$ be the time when information about the referendum result becomes known to the market. There are two possible states, Leave (L) and Remain (R). Betting payoffs at time $\tau$ can be related to state prices $\psi_{\mathrm{L}}\left(\psi_{\mathrm{R}}\right)$ at time $t<\tau$, i.e., the prices of the Arrow securities paying off 1 in case of state Leave (Remain) and 0 otherwise (see, e.g., Duffie (2001, Chapter 1), or LeRoy and Werner (2014, Sections 5.2 and 5.7)). Throughout this paper, we assume constant and flat interest rates and the existence of a unique riskneutral pricing measure in each currency. For bets placed in U.S. dollars, the state prices in dollars can be computed as the payoffs' discounted expected values under the standard risk-neutral measure $\mathbb{Q}^{\$}$, which uses the dollar money market account as the numeraire:

$$
\begin{aligned}
\psi_{\mathrm{L}}^{\$} & =e^{-r(\tau-t)} \mathbb{E}_{t}^{\$}\left[1_{\mathrm{L}}\right] \\
& =e^{-r(\tau-t)} q_{\mathrm{L}}^{\$},
\end{aligned}
$$


where $r$ is the (constant) U.S. risk-free interest rate, $1_{\mathrm{L}}$ denotes the indicator function taking on a value of 1 (corresponding to a payoff of one U.S. dollar) if the referendum outcome is Leave and 0 otherwise, and we suppress the time subscripts for state prices and risk-neutral probabilities for notational simplicity. $q_{\mathrm{L}}^{\$}$ is usually referred to as the "risk-neutral Leave probability" under the measure $\mathbb{Q}^{\$}$ (see, e.g., Duffie 2001, Chapter 1), or LeRoy and Werner (2014. Sections 5.2 and 5.7)), and $q_{\mathrm{R}}^{\$}$ is defined analogously. The term "risk-neutral valuation" is used also in the case of incomplete markets, where the particular "risk-neutral" probability measure chosen by the market contains a risk premium (see, e.g., Björk, 2009, Section 15.2). Equation (1) directly links state prices and risk-neutral event outcome probabilities. This relation must hold for any currency. For bets placed in pounds, the relation between state price and risk-neutral Leave probability reads as

$$
\begin{aligned}
\psi_{\mathrm{L}}^{£} & =e^{-i(\tau-t)} \mathbb{E}_{t}^{£}\left[1_{\mathrm{L}}\right] \\
& =e^{-i(\tau-t)} q_{\mathrm{L}}^{£},
\end{aligned}
$$

where $i$ is the U.K. risk-free interest rate.

Odds quoted by bookmakers or on betting exchanges follow different market conventions, with fractional odds and decimal odds being the most common. Their conversion into state prices is straightforward. Fractional odds of 4/1 mean that for every unit you bet, winning results in a gain of 4 units, plus you get your stake back for a total payoff of 5 . The equivalent decimal odds would be 5.0, describing the total winning payoff for a stake of 1 . Both odds correspond to a state price of 0.2 . For positive risk-free interest rates, equations (1) and (2) imply risk-neutral probabilities slightly above 0.2 , depending on the remaining time to the event. In practice, given that bets usually have short maturities, interest rates are frequently ignored. Odds quoted in betting markets usually include a bid-ask spread, which in the context of betting is called the "cut". Because of the cut, the sum of state 
prices across all event outcomes (even without discounting, i.e., immediately before the event) is higher than 1, and savvy punters adjust for the cut when converting betting odds into implied outcome probabilities.

For events whose payoffs are orthogonal to the pricing kernel, betting odds should not carry a risk premium. Put differently, for such events realworld probabilities and risk-neutral probabilities should be equal in theory. This holds for most events on which bets are placed, e.g., for sports events. For this reason, (cut-adjusted) odds of $4 / 1$ in fractional notation or 5.0 in decimal notation would be usually interpreted by market participants as implying a real-world outcome probability of 0.2 .4 For political events with effects on economic variables, non-zero risk premia in betting odds should be expected from basic financial economics theory. This implies that risk-neutral outcome probabilities for such events should differ from the corresponding real-world outcome probabilities. In equations (1) and (2), we use different symbols for the risk-neutral Leave probabilities depending on the numeraire. In what follows, we will show that for events affecting exchange rates, not only do risk-neutral probabilities for different event outcomes differ from real-world probabilities, but risk-neutral probabilities themselves differ across currencies, i.e., they depend on the particular probability measure used. This implies that betting odds in different currencies should differ even if interest rates in these currencies were identical. This is in contrast to events that are unrelated to exchange rates: For instance, the risk-neutral probabilities for the possible outcomes of a soccer game between Italy and Brazil should be identical under measures $\mathbb{Q}^{\$}$ and $\mathbb{Q}^{£}$.

\subsection{Exchange Rates under Different Measures}

In this section, we present two variants of our model for the reactions of exchange rate to major events. We start out with deterministic conditional

\footnotetext{
${ }^{4}$ This corresponds to common explanations of betting odds, see, e.g., https:// tinyurl.com/y9a4rfxm.
} 
returns depending on the event outcome, which will then be generalized to stochastic conditional returns in a mixture of densities model in Section 2.2.2.

\subsubsection{Deterministic Conditional Returns}

We denote by $S_{t}$ the GBPUSD exchange rate, which uses the USD as the numeraire, and by $d(u)$ the down (up) factor defined by $S_{\tau} / S_{t}$ in case of Leave (Remain). The expected GBPUSD exchange rate under $\mathbb{Q}^{\$}$ must be equal to the forward exchange rate. Assuming that the event outcome does not depend on the exchange rate (i.e., that the development of the GBPUSD rate before the referendum does not affect the voting behavior), we get

$$
\begin{aligned}
e^{(r-i)(\tau-t)} S_{t} & =\mathbb{E}_{t}^{\$}\left[S_{\tau}\right] \\
& =S_{t} \mathbb{E}_{t}^{\$}\left[d 1_{\mathrm{L}}+u 1_{\mathrm{R}}\right] \\
& =S_{t}\left[d q_{\mathrm{L}}^{\$}+u\left(1-q_{\mathrm{L}}^{\$}\right)\right] .
\end{aligned}
$$

This martingale condition is equivalent to

$$
d q_{\mathrm{L}}^{\$}+u\left(1-q_{\mathrm{L}}^{\$}\right)=e^{(r-i)(\tau-t)}
$$

Along the same lines, we get for the USDGBP rate, which uses the pound as numeraire 5

$$
\begin{aligned}
e^{(i-r)(\tau-t)} \frac{1}{S_{t}} & =\mathbb{E}_{t}^{£}\left[\frac{1}{S_{\tau}}\right] \\
& =\frac{1}{S_{t}}\left[\frac{1}{d} \mathbb{E}_{t}^{£}\left[1_{\mathrm{L}}\right]+\frac{1}{u}\left(1-\mathbb{E}_{t}^{£}\left[1_{\mathrm{L}}\right]\right)\right] \\
& =\frac{1}{S_{t}}\left[\frac{q_{\mathrm{L}}^{£}}{d}+\frac{1-q_{\mathrm{L}}^{£}}{u}\right],
\end{aligned}
$$

${ }^{5}$ For a discussion of exchange rate processes $S_{t}$ and $1 / S_{t}$ under the corresponding measures used here, see Hull (2018, Section 30.3). 
which can be rewritten as

$$
\frac{q_{\mathrm{L}}^{£}}{d}+\frac{1-q_{\mathrm{L}}^{£}}{u}=e^{(i-r)(\tau-t)}
$$

The solution to the system of equations (4) and (7) is

$$
d=\frac{q_{\mathrm{L}}^{£}}{q_{\mathrm{L}}^{\Phi}} e^{(r-i)(\tau-t)}=\frac{\psi_{L}^{£}}{\psi_{L}^{\Phi}}, \quad u=\frac{1-q_{\mathrm{L}}^{£}}{1-q_{\mathrm{L}}^{\Phi}} e^{(r-i)(\tau-t)}=\frac{\psi_{R}^{£}}{\psi_{R}^{\$}} .
$$

Equation (8) relates the conditional returns $d$ and $u$ to the risk-neutral Leave and Remain probabilities under both risk-neutral measures. For events that do not affect the GBPUSD exchange rate (e.g., a soccer game between Italy and Brazil), $d=u=\exp [(r-i)(\tau-t)]$, which implies $q_{\mathrm{L}}^{£}=q_{\mathrm{L}}^{\$}$. As intuition suggests, the risk-neutral probabilities for such events are identical across numeraires, i.e., they are the same for both currencies. Note that state prices and, hence, betting odds, may still differ non-negligibly for such events whenever $r \neq i$ and $(\tau-t)$ is large. For events whose outcomes affect the exchange rate, however, our model implies that the risk-neutral event probabilities should differ.

\subsubsection{Stochastic Conditional Returns}

The derivations in Section 2.2.1 still hold in case of stochastic conditional returns, which we denote by $\tilde{d}$ and $\tilde{u}$. E.g., equation (3) becomes

$$
\begin{aligned}
e^{(r-i)(\tau-t)} S_{t} & =\mathbb{E}_{t}^{\$}\left[S_{\tau}\right] \\
& =S_{t} \mathbb{E}_{t}^{\$}\left[\tilde{d} 1_{\mathrm{L}}+\tilde{u} 1_{\mathrm{R}}\right] \\
& =S_{t}\left[\bar{d} q_{\mathrm{L}}^{\$}+\bar{u}\left(1-q_{\mathrm{L}}^{\$}\right)\right],
\end{aligned}
$$


where $\bar{d}$ and $\bar{u}$ are the expected values of $\tilde{d}$ and $\tilde{u}$, respectively. The martingale condition (4) becomes

$$
\bar{d} q_{\mathrm{L}}^{\$}+\bar{u}\left(1-q_{\mathrm{L}}^{\$}\right)=e^{(r-i)(\tau-t)},
$$

and analogous changes apply to equations (6) and (7). For stochastic exchange rate reactions to events, equation (8) then reads as

$$
\bar{d}=\frac{q_{\mathrm{L}}^{£}}{q_{\mathrm{L}}^{\$}} e^{(r-i)(\tau-t)}=\frac{\psi_{L}^{£}}{\psi_{L}^{\Phi}}, \quad \bar{u}=\frac{1-q_{\mathrm{L}}^{£}}{1-q_{\mathrm{L}}^{\$}} e^{(r-i)(\tau-t)}=\frac{\psi_{R}^{£}}{\psi_{R}^{\$}} .
$$

It now relates the expected conditional returns $\bar{d}$ and $\bar{u}$ to the risk-neutral Leave and Remain probabilities under both risk-neutral measures.

As a specific example for the dynamics of exchange rates around major events, we use a model that is inspired by Hanke et al. (2019). It is rooted in the literature on option-implied risk-neutral densities (an overview of different estimation methods is provided by Figlewski, 2010). One approach to modeling risk-neutral return densities uses mixtures of parametric densities. Ritchey (1990) shows that by mixing two or three normal densities, a wide range of empirically observed skewness and kurtosis values can be generated for the combined densities while still retaining analytical tractability. Hanke et al. (2019) apply this model to exchange rates after major events, and give the model an economic meaning by interpreting the component densities as conditional return densities, with the weights corresponding to the risk-neutral event outcome probabilities estimated from betting odds. For the Brexit referendum, this means that exchange rates immediately after the referendum (at time $\tau$ ) are assumed to be conditionally lognormal, with parameters of these conditional densities depending on the event outcome.

We denote by $\phi_{t, \tau}^{\prime}(\cdot)$ the density of $\log$ returns, $\ln \left(S_{\tau} / S_{t}\right)$, under the riskneutral measure $\mathbb{Q}^{\$}$. Denoting by $\ln \tilde{d}(\ln \tilde{u})$ the conditional $\log$ returns of $S$ from $t$ to $\tau$ in case of Leave (Remain), we define their conditional densities 
as

$$
\begin{aligned}
& \ln \tilde{d} \sim N\left(\mu_{L}^{\$}(\tau-t), \sigma_{L} \sqrt{\tau-t}\right), \\
& \ln \tilde{u} \sim N\left(\mu_{R}^{\$}(\tau-t), \sigma_{R} \sqrt{\tau-t}\right) .
\end{aligned}
$$

The combined risk-neutral density is given as a mixture of these conditional normal densities (cf. also Figure 1):

$$
\begin{aligned}
\phi_{t, \tau}^{\prime}(\cdot) & =q_{\mathrm{L}}^{\$} \phi_{t, \tau}^{\prime}(\cdot \mid \mathrm{L})+q_{\mathrm{R}}^{\$} \phi_{t, \tau}^{\prime}(\cdot \mid \mathrm{R}) \\
& =q_{\mathrm{L}}^{\$} N\left(\mu_{L}^{\$}(\tau-t), \sigma_{L} \sqrt{\tau-t}\right)+q_{\mathrm{R}}^{\$} N\left(\mu_{R}^{\$}(\tau-t), \sigma_{R} \sqrt{\tau-t}\right) .
\end{aligned}
$$

Hanke et al. (2019) estimate the parameters of these conditional distributions by combining information from betting odds and one-month/three-months option prices that expire on the day after the respective events. Figure 1 uses the parameters they estimated just less than one month before the referendum. For this mixture of normals model, $\bar{d}$ in equations (9)-(11) is given by $\bar{d}=\exp \left[\left(\mu_{L}^{\$}+0.5 \sigma_{L}^{2}\right)(\tau-t)\right]$ ( $\bar{u}$ is defined analogously).

Hanke et al. (2019) derive conditional exchange rate forecasts by combining risk-neutral outcome probabilities from betting odds with implied risk-neutral densities from FX option prices. We note that if observable betting odds were unbiased and already incorporated the effect described in equation (11), the outcome-dependent conditionally expected returns in the exchange rates could be directly inferred from the ratios of the implied riskneutral outcome probabilities under different numeraires, without any need for FX option price data. As long as bookmakers quote identical odds across transaction currencies, however, this is not possible. 


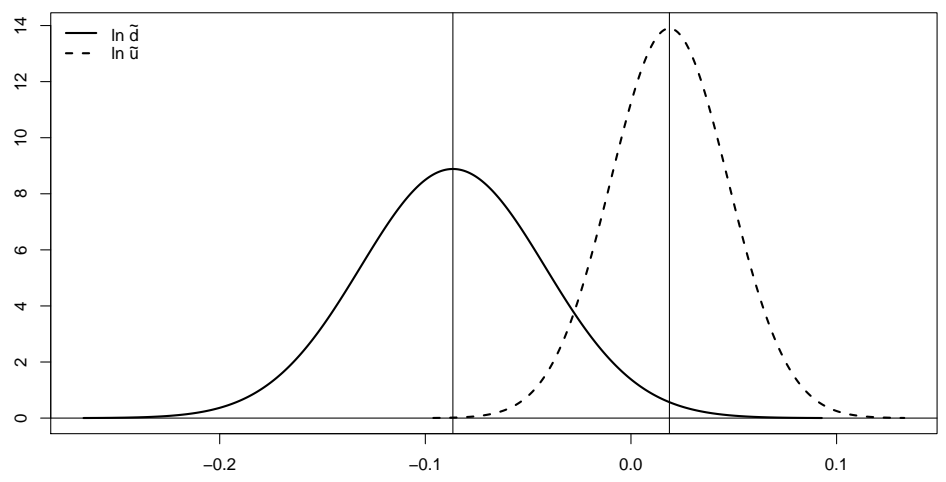

Figure 1: Conditional return densities for the possible outcomes Leave and Remain. Parameter values are taken from Hanke et al. (2019, Table 2) and have been estimated from one-month GBPUSD options and betting odds, both observed just less than one month before the referendum.

\section{Consequences of Equal Betting Odds across Currencies}

Equation (11) shows that risk-neutral probabilities should differ across currencies for events whose outcomes affect exchange rates. For both events described in the introduction, however, betting odds quoted by all major bookmakers and betting exchanges were identical for all transaction currencies offered on the respective websites. In this section, we analyze the consequences of such mispricing under two different assumptions: In Section 3.1, we start from the assumption of deterministic outcome-dependent conditional returns introduced in Section 2.2.1. This simplified setting allows us to gain valuable insights by deriving no-arbitrage conditions. Based on these results, we then show in Section 3.2 that the corresponding arbitrage strategies turn into good deals or approximate arbitrage opportunities under the assumption of stochastic conditional returns, which was analyzed 
in Section 2.2.2.

\subsection{Deterministic Conditional Returns: Arbitrage Op- portunities}

Equation (8) shows how the ratios of risk-neutral event outcome probabilities under different numeraires are linked to $u$ and $d$. Although betting odds in affected currencies should reflect these relations, they were identical across transaction currencies for both the Brexit referendum and the U.S. presidential elections. In this section, we derive no-arbitrage conditions that apply in this situation.

Denote the state prices available in betting markets for the Brexit example by $p_{L}^{\$}\left(p_{L}^{£}\right)$. These empirical analogues to the state prices $\psi_{L}$ can be computed from betting odds using the conventions described in Section 2.1. Assume that we are at time $\tau^{-}$, i.e., immediately before the event, so that $p_{L}=q_{L}$ in both currencies (cf. equations (1) and (2) $)^{6}$ and $\exp [(r-i)(\tau-t)]=$ 1. Without loss of generality, assume that $d<1<u$, and contrary to equation (8), but in line with odds quoted on major websites, $p_{L}^{\$}=p_{L}^{£}$. For the moment, we assume there are no transaction costs for buying/selling foreign currency. To begin with the simplest case, assume further that there is no cut, so $p_{R}=1-p_{L}$ in both currencies. Equation (8) implies that in this situation the Leave odds are overpriced in pounds relative to the dollar, while the opposite holds for the Remain odds. Therefore, a simple arbitrage strategy can be constructed $\sqrt[7]{7}$ which starts with zero wealth. This strategy will be referred to as Strategy 1 in what follows. We borrow 1 pound, bet $p_{R}^{£}$ pounds on Remain, convert the rest into dollars, and bet the resulting amount of $\left(1-p_{R}^{£}\right) S_{\tau^{-}}$dollars on Leave. Stakes and outcome-dependent payoffs are shown in Figure 2. For a stake of 1 pound, we get an immediate

\footnotetext{
${ }^{6}$ Identical results would obtain for $t<\tau$ and $r=i=0$.

${ }^{7}$ This strategy is similar to the one suggested by Neuberger (2016), which uses euros instead of dollars.
} 


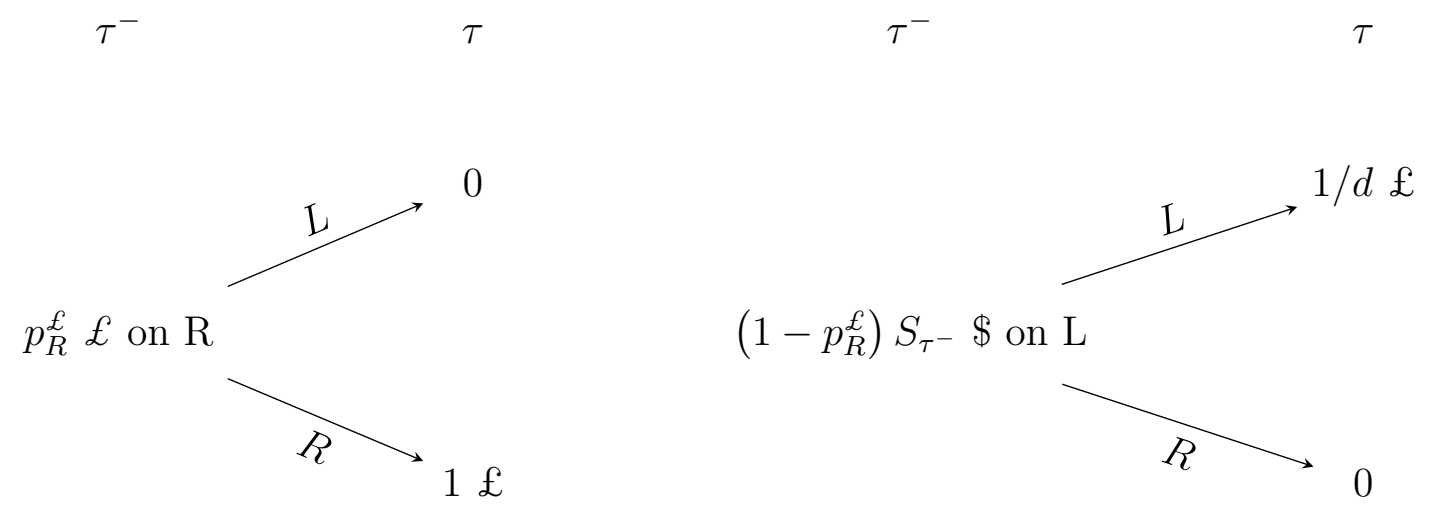

Figure 2: Strategy 1 - stakes and payoffs for the two bets involved (left: bet in pounds, right: bet in dollars). Stakes and payoffs are given assuming no cut, i.e., $c=0$ ), and before repayment of the $1 £$ borrowed initially.

payoff of 1 pound in case of Remain, and - converting back after the move of the exchange rate to $S_{\tau}=d S_{\tau^{-}}$- a payoff of $(1 / d)$ pounds in case of Leave. This is an arbitrage because of $d<1$.

If we assume that bookmakers take an identical cut in both currencies equal to $c=p_{L}+p_{R}-1$, following Strategy 1 and betting $p_{R}^{£}$ pounds on Remain leaves only $\left(p_{L}^{£}-c\right) S_{\tau^{-}}$dollars to bet on Leave. The reduced stake compared to the case without a cut leads to a proportional reduction in the payoff to be received if Leave occurs. This reduction may be outweighed by a sufficiently large move in the exchange rate. Strategy 1 remains an arbitrage if (after conversion into pounds, and remembering that by assumption $p_{L}^{£}=$ $\left.p_{L}^{\$}\right)$

$$
\begin{gathered}
{\left[1-\frac{c}{p_{L}^{\$}}\right] \frac{1}{d}>1} \\
1-\frac{c}{p_{L}^{\$}}>d .
\end{gathered}
$$


Using equation (8), this leads to

$$
q_{L}^{\$}-q_{L}^{£}>c \frac{q_{L}^{\$}}{p_{L}^{\$}}
$$

If betting odds in dollars available in the market differ from their theoretical values only because of the cut, three cases are possible: (i) $p_{L}^{\$}$ is correct and $p_{R}^{\$}$ is overpriced by $c$, (ii) $p_{R}^{\$}$ is correct and $p_{L}^{\$}$ is overpriced by $c$, (iii) both $p_{L}^{\$}$ and $p_{R}^{\$}$ are overpriced so that $p_{L}^{\$}+p_{R}^{\$}-1=c$. For case (i), we get from equation 15

$$
q_{L}^{\$}-q_{L}^{£}>c .
$$

In the other cases, the ratio on the right-hand side of equation (15) will be smaller than 1. For typical sizes of the cut $c$ observed in practice, however, the difference will be relatively small, so that equation $(16)$ is a good approximation that errs on the conservative side: If equation 16 indicates an arbitrage opportunity, then inequality (15) is satisfied as well. Equation (14) provides a no-arbitrage condition based on quantities that are either observable in the market $\left(c, p_{L}\right)$ or assumed to be known in our model $(d)$. Equations (15) and (16) provide simpler relations based on the theoretically correct risk-neutral probabilities, which cannot be inferred precisely from betting odds available in the market if $c>0$, however.

Alternatively, a similar strategy ("Strategy 2") can be followed that starts by borrowing 1 dollar instead of 1 pound. Betting $p_{L}^{\$}$ dollars on Leave, converting the rest into pounds and betting it on Remain leads to the following conditions, which are symmetric to equations (14) to (15):

$$
\begin{gathered}
1-\frac{c}{p_{R}^{£}}>\frac{1}{u} \\
q_{R}^{£}-q_{R}^{\$}>c \frac{q_{R}^{£}}{p_{R}^{£}} .
\end{gathered}
$$

If, in analogy to (but different from) case (ii) described near equation (15), 
the Remain odds in pounds are correct, we get

$$
q_{R}^{£}-q_{R}^{\$}>c .
$$

When comparing the no-arbitrage conditions in (14)-(16), which result from Strategy 1, to the conditions in (17)-(19), which result from Strategy 2, we note the following: First, whereas conditions (14)-(15) are symmetric to conditions (17)-(18), they are not equivalent. Hence, there are cases where an arbitrage strategy can be set up starting from one currency, but not from the other. This will be illustrated numerically in Section 4. Second, while equations (16) and (19) are equivalent, they have been derived under different assumptions: Equation (16) holds if the Leave odds in dollars are correct, while equation (19) holds if the Remain odds in pounds are correct.

Table 1 summarizes the payoffs associated with our two arbitrage strategies for the base case, with and without a cut. If condition (14) holds, Strategy 1 yields a positive profit (payoff higher than stake) in case of Leave, and zero profit in case of Remain. If condition (17) holds, Strategy 2 yields a positive profit in case of Remain, and zero profit in case of Leave. In what follows, we show that if and only if both conditions hold, it is possible to construct combinations of both strategies, which provide a positive profit in each of the states. To this end, we invest $y$ pounds in Strategy 1 (the "pound leg" of our combined strategy) and $z$ dollars in Strategy 2 (the "dollar leg"). Setting $z=(1-y) S_{\tau^{-}}$normalizes the total amount at stake to the equivalent of 1 pound. The profit on the pound leg is either zero (in case of Remain) or

$$
\operatorname{profit}_{L}^{\mathcal{L}}=y\left[1-\frac{c}{p_{L}^{\Phi}}\right] \frac{1}{d}-y
$$

(in case of Leave, cf. equation (14)). Similarly, the profit on the dollar leg is 


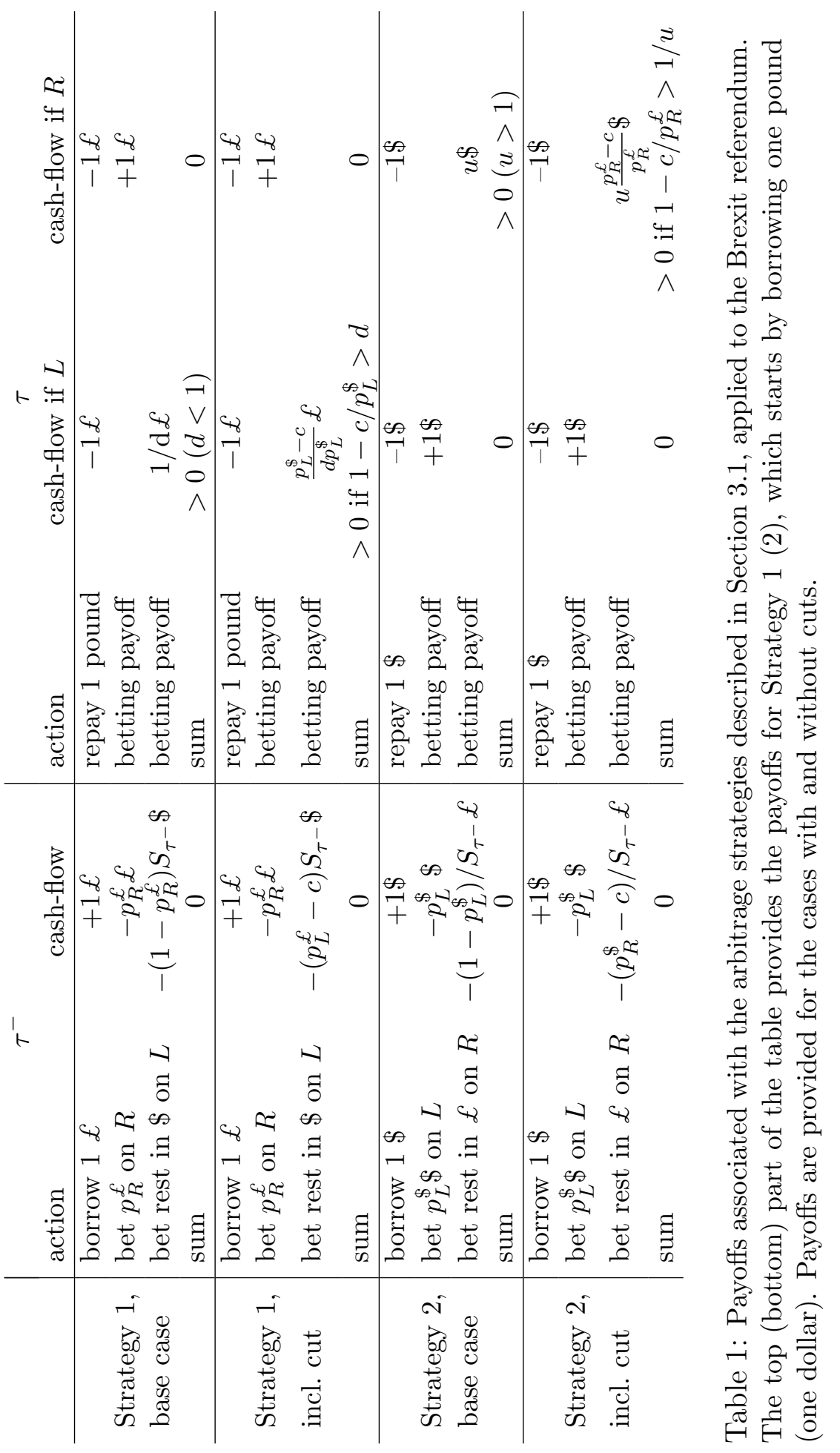


either zero (in case of Leave) or

$$
\operatorname{profit}_{R}^{\$}=z\left[1-\frac{c}{p_{R}^{£}}\right] u-z
$$

(in case of Remain, cf. equation (17)). Converting this into pounds requires dividing by the exchange rate, which is $S_{\tau}=u S_{\tau^{-}}$in this case. Hence, the profit on the combined strategy, expressed in pounds, is

$$
\text { profit }^{£}=\left\{\begin{array}{ll}
y\left(\left[1-\frac{c}{p_{L}^{\Phi}}\right] \frac{1}{d}-1\right) & \text { Leave } \\
z\left(\left[1-\frac{c}{p_{R}^{\complement}}\right] u-1\right) /\left(u S_{\tau^{-}}\right) & \text {Remain }
\end{array} .\right.
$$

The payoff in case of Leave (Remain) is positive if and only if condition (14) (condition (17)) holds. This shows that combinations of the strategies yielding positive profits in both states are possible if and only if both conditions hold. In this case, it is even possible to construct "risk-free" arbitrage strategies with equal profits in both states. The required amount $y^{*}$ to invest in the pound leg of the combined strategy can be calculated by equating the profits in both states given in equation (22) and solving for $y$ :

$$
y^{*}=\frac{\left[1-\frac{c}{p_{R}^{\complement}}\right]-\frac{1}{u}}{\left[1-\frac{c}{p_{R}^{\complement}}\right]-\frac{1}{u}+\left[1-\frac{c}{p_{L}^{\Phi}}\right] \frac{1}{d}-1} .
$$

The corresponding dollar amount to be invested in the dollar leg is then $z^{*}=\left(1-y^{*}\right) S_{\tau^{-}}$. Along the same lines, a strategy providing equal dollar profits in both states can be constructed, since both arbitrage conditions need to be satisfied. As noted above, the resulting strategy is symmetric to that yielding equal pound profits across states, but not equivalent.

In reality, buying and selling of foreign currency involves transaction costs. Assuming transaction costs $\gamma$ that are proportional to the transaction amount, Strategy 1 incurs transaction costs (in pounds, cf. Table 1 ) 
of

$$
\gamma\left(1-p_{R}^{£}+\frac{1}{d}\left(p_{L}^{\$}-c\right) /\left(p_{L}^{\$}\right)\right)
$$

while Strategy 2 incurs transaction costs (in dollars) of

$$
\gamma\left(1-p_{L}^{\$}+u\left(p_{R}^{£}-c\right) / p_{R}^{£}\right)
$$

For an arbitrage opportunity to exist, the differences between the two sides of the inequalities derived above would have to be large enough to cover these transaction costs.

\subsection{Stochastic Conditional Returns: Good Deal/Approximate Arbitrage Opportunities}

As already noted by Neuberger (2016), we could not have completely ruled out a strengthening of the pound after a majority voting for Leave: The probability of such a reaction by financial markets may have been small, but certainly not zero. In this section, we analyze the results of the strategies discussed above for the assumption of stochastic outcome-dependent conditional returns $\tilde{u}$ and $\tilde{d}$ (cf. Section 2.2.2). The empirical data we will use to illustrate this case in Section 4.2 are estimated from one-month options. For this reason, we consider now bets placed at time $t<\tau^{-}$in the presence of identical odds across currencies. In light of our derivations in Section 2.2, a good time for placing a bet on a certain outcome would be when the betting odds for this outcome are very low. Then, today's exchange rate, which is the discounted risk-neutral expected value of the exchange rate after the event, gives rise to large conditionally expected returns. From equation (11), we see that differences in the risk-free interest rates may slightly dampen or amplify the observed mispricing, which results from equal betting odds across currencies. For most currencies, however, this difference is only of second order to the effect we describe, particularly in the light of the relatively short 
maturities of most bets. In order to focus on our main message, we therefore assume $r=i=0$ and a cut of 0 in what follows.

In the presence of stochastic conditional returns the strategies described in the previous section are no longer arbitrage opportunities, but may still be good deals in the sense of Cochrane and Saa-Requejo (2002), i.e., they may deliver high Sharpe ratios. The results in Section 4.2 will show that for the two empirical cases analyzed there, they are even approximate arbitrage opportunities in the sense of Shanken (1992). We reconsider Strategy 1 described in Section 3.1, which starts by borrowing 1 pound and is illustrated in Figure 2. In contrast to Section 3.1, however, we implement the strategy now at time $t<\tau$. In the absence of a cut and for deterministic conditional returns, this strategy gives rise to arbitrage opportunities whenever $d<1$. Hence, for a stochastic downward move of size $\tilde{d}$, the strategy is only profitable if the realized return is smaller than 1 . The probability for observing a realization smaller than 1 depends on the parameters of its distribution: The higher $\left|\mu_{L}^{\mathbb{P}} / \sigma_{L}\right|$ (where $\mu_{L}^{\mathbb{P}}$ is the corresponding parameter of the density of $\tilde{d}$ under the real-world probability measure $\mathbb{P}$; note that $\mu_{L}<0$ is necessary for $\bar{d}<1$ ), the higher this probability will be.

The (log) return on Strategy 1 is given by

$$
\tilde{r}_{1}=\left\{\begin{array}{ll}
0 & \text { with probability } q_{R}^{\mathbb{P}} \\
-\ln \tilde{d} & \text { with probability } q_{L}^{\mathbb{P}}
\end{array},\right.
$$

where $q_{L}^{\mathbb{P}}$ and $q_{R}^{\mathbb{P}}$ denote the real-world event probabilities. To assess the strategy's attractiveness, any standard approach may be used, such as expected utility or risk-adjusted performance measures like the Sharpe ratio:

$$
S R_{1}=\frac{\mathbb{E}_{t}^{\mathbb{P}}\left[\tilde{r}_{1}\right]}{\sigma_{\tilde{r}_{1}} \sqrt{\tau-t}}=-\frac{q_{L}^{\mathbb{P}} \mathbb{E}_{t}^{\mathbb{P}}[\ln \tilde{d}]}{q_{L}^{\mathbb{P}} \sigma_{L}(\tau-t)}=-\frac{\mu_{L}^{\mathbb{P}}}{\sigma_{L}}
$$

where $S R_{1}$ is the annualized Sharpe ratio of Strategy 1 , and $\mathbb{E}_{t}^{\mathbb{P}}[\cdot]$ denotes 
time $t$ expectation under the real-world measure. Equation 27) shows that in addition to the probability for a positive payoff of Strategy 1 being, ceteris paribus, increasing in $\left|\mu_{L}^{\mathbb{P}} / \sigma_{L}\right|$, this expression is also the strategy's Sharpe ratio. Estimates for the volatility $\sigma_{L}$ and the risk-neutral expected returns $\mu_{L}^{\$}$ and $\mu_{L}^{£}$ can be obtained using the methodology described in Hanke et al. (2019). Adding an estimated risk premium to the risk-neutral expected return yields an estimate for $\mu_{L}^{\mathbb{P}}$. In the literature (see Figure 3 in Jurek and $\mathrm{Xu}, 2014)$, we find an implied risk premium for the GBPUSD rate of around $2 \%$. Given that implied at-the-money volatilities of 1-month options in the run-up to the Brexit referendum were around 2-3 times higher than in normal times (see Figure 3 in Hanke et al., 2019), we use a conservatively high risk premium of $6 \%$ per year for the GBPUSD pair in our calculations. For the USDMXN rate, Jurek and $\mathrm{Xu}(2014)$ do not report a risk premium. The risk premium range they provide in their Figure 3 for 24 currency pairs is between $-6 \%$ and $6 \%$ during normal times, with most values between $-4 \%$ and $4 \%$. From Hanke et al. (Figure 3 in 2019), we see that 1-month implied volatilities for USDMXN options in the run-up to the U.S. presidential elections were around twice as high as usual, leading us to a conservatively high risk premium estimate of around $12 \%$ per year. Our numerical results in Section 4 will show that even with these conservative risk premium estimates, Strategy 1 yields very favorable Sharpe ratios. For Strategy 2, similar considerations apply.

\section{Numerical Illustration}

In this section, we illustrate our findings from Section 3 numerically. Following the structure of that section, we start by assuming deterministic conditional returns after events and illustrate the resulting no-arbitrage conditions. Then, we consider the effect of typical cuts observed in the market, using real-world betting odds that were available for the Brexit referendum 
and the U.S. presidential elections. Finally, we illustrate the stochastic case using estimated conditional exchange rate distributions.

\subsection{Deterministic Conditional Returns}

For both events mentioned in the introduction, Hanke et al. (2019, Section IV.A) report realized exchange rate moves (GBPUSD and USDMXN) of around $10 \%$, with precise returns depending on the exact date before the event that is used for comparison. In their Section $\mathrm{V}$, they show that these changes could have been forecast quite accurately from observable betting odds and FX options prices. For someone who ignores the uncertainty in the conditional return and interprets this number as deterministic, a downward movement in the GBPUSD rate of $10 \%$ in case of Leave corresponds to $d=0.9$ in our notation. Risk-neutral Leave probabilities implied from betting odds were between 0.2-0.4, and risk-neutral Trump probabilities were between 0.1-0.35. To get a first indication whether arbitrage opportunities

might have existed in practice under this assumption, we plug these values into equation (11) to see by how much risk-neutral probabilities immediately before the events should have differed between the two currencies in theory. From equation (11), we get that the differences between risk-neutral Leave (Trump) probabilities under the respective numeraires should have been approximately 2-4 (1-3.5) percentage points. Equations (16) and (19) show that cuts below these ranges would not have been enough to make these arbitrage opportunities vanish.

For a more precise calculation, we choose plausible odds that lead to riskneutral outcome probabilities in the ranges indicated above. We deliberately choose values that illustrate numerically the case mentioned in Section 3.1 . where Strategy 1 is an arbitrage, but Strategy 2 is not. Assume that observed Leave odds are 5.36 in decimal notation, and observed Remain odds are 1.2. Out of ignorance regarding the effect described in this paper, the odds available in the market are identical across transaction currencies. Assume 
that $d=0.9$ and $u=1.03$. Converting the odds to state prices yields $p_{L}^{\$}=p_{L}^{£}=0.1866, p_{R}^{\$}=p_{R}^{£}=0.8333$, and a cut of almost exactly $2 \%$ : $c=p_{L}+p_{R}-1=0.0199$. In this situation, the arbitrage condition (17) is satisfied, as

$$
\begin{aligned}
1-\frac{0.0199}{0.8333} & >1 / 1.03 \\
0.976 & >0.971,
\end{aligned}
$$

but the arbitrage condition (14) is violated:

$$
\begin{aligned}
1-\frac{0.0199}{0.1866} & \ngtr 0.9 \\
0.893 & \ngtr 0.9 .
\end{aligned}
$$

In the example, we assumed a cut of approximately $2 \%$. In practice, punters may either place their bets with bookmakers directly, or they may channel their bets through a betting exchange. When dealing with bookmakers directly, punters can only accept odds quoted by the bookmaker - in betting jargon, they can only "back" a bet offered by the bookmaker. The bookmaker quotes odds that imply a cut of around 2-8\% to cover his costs and make a profit. On betting exchanges, punters can both back bets that are offered by others, and they can offer bets themselves, which is referred to as "laying" a bet. The latter is analogous to going short in financial markets. The business model of betting exchanges generates profits not from the cut, which is typically much smaller than bookmakers' cuts, but from commissions, which usually have to be paid by the winning side of a bet. These commissions are on the order of $5 \%$ and may be lower for punters who generate a lot of transactions on the platform. On Betfair Exchange, the largest platform, the minimum commission (reserved for prime customers who generate a lot of business on the platform) is $2 \%$ at the time of writing, which must be added to the bid-ask spread of usually around $0-0.5 \%$ to cal- 
culate total transaction costs. When implementing the strategies described in Section 3.1 on betting exchanges, the commissions they charge provide a lower bound on transaction costs. This is not the case when placing the respective bets with online bookmakers: Since odds differ across bookmakers, these differences in odds can be exploited to reduce the cut that effectively applies by simply dealing with different bookmakers for the two legs of the strategy.

Comparisons of odds are simplified by dedicated websites, which offer real-time odds across major bookmakers free of charge. At the time of writing, www.oddschecker.com is one of the leading odds comparison sites. To get historical data from this website, the "Wayback Machine" at web. archive.org can be used, which provides snapshots of websites taken at irregularly spaced time points in the past (on average every 2-4 days for www.oddschecker.com in 2016). Table 2 provides bookmakers' odds from several large bookmakers for the Brexit referendum, which were retrieved in this way for June 16, 2016, one week before the referendum. The table

\begin{tabular}{lcccccc} 
& Paddy Power & Stan James & 888 Sport & Ladbrokes & William Hill & min \\
\hline Remain & $8 / 15$ & $8 / 13$ & $6 / 11$ & $8 / 15$ & $1 / 2$ & \\
Leave & $6 / 4$ & $6 / 5$ & $16 / 11$ & $6 / 4$ & $13 / 8$ & \\
\hline Remain & 0.652 & 0.619 & 0.647 & 0.652 & 0.667 & 0.619 \\
Leave & 0.4 & 0.455 & 0.407 & 0.4 & 0.381 & 0.381 \\
\hline Cut (in \%) & 5.22 & 7.36 & 5.45 & 5.22 & 4.76 & 0.00
\end{tabular}

Table 2: Top: Betting odds for the Brexit referendum from selected major bookmakers. Bottom: State prices and cuts calculated from these betting odds. Rightmost column: Best quotes (viewed separately) across bookmakers for Remain and Leave and resulting effective cut. Source: www.oddschecker.com, archived at www.webarchive.org on June 16, 2016, $14: 52$.

shows in the upper part odds across various bookmakers. In the lower part, we converted the betting odds to state prices (ignoring the negligible interest rate difference between these two currencies for one week) and calculated the cut implied in these odds. The rightmost column shows the minimum state 
prices across bookmakers, together with the effective minimum cut a punter could have achieved by using different bookmakers for the two legs of the strategy. If this minimum cut had been negative, simple arbitrage strategies would have been possible with both legs in the same currency. Bookmakers are well aware of these simple arbitrage strategies and usually take other bookmakers' odds into account when deciding which odds to offer. In spite of this, the existence of arbitrage opportunities has been documented in the literature, see e.g. Franck et al. (2013) for inter-market arbitrage using bets placed with bookmakers and betting exchanges. Ensuring that this implied cut is never negative protects bookmakers from being exploited via simple arbitrage strategies.

In the presence of the effect described in this paper, however, ensuring a minimum cut of zero across bookmakers no longer provides protection against arbitrage. In Table 2, the best available Remain odds of 0.619 were offered by Stan James, while the best available Leave odds of 0.381 were offered by William Hill, implying a cut of exactly 0 . The simple arbitrage we devised in Section 3.1 in the absence of a cut (Strategy 1) called for betting on Remain in pounds and betting on Leave in dollars. To ensure that this strategy is feasible, we check the respective bookmakers' websites for the transaction currencies they offer, confirming that Stan James does offer pounds and William Hill does offer dollars as transaction currencies. Putting a stake of 0.619 pounds on Remain with Stan James, converting the rest into dollars, and betting $0.381 S_{\tau^{-}}^{\text {GBPUSD }}$ dollars on Leave with William Hill would have resulted in a payout of 1 pound in case of Remain and $1 / d>1$ pounds after converting back from dollars. In the absence of transaction costs, the only condition required for a profit in case of Leave is $d<1$, i.e., a weakening of the pound in case of Leave, and the correct anticipation of this effect. For the alternative outcome of Remain, the strategy protects against any losses.

For the U.S. presidential elections, a similar example can be provided. Following the derivation in Section 2, which used the terminology of the 
Brexit case, we note that the analogue to the GBPUSD rate in terms of notation is the (lesser used) MXNUSD rate, so the peso takes on the role of the pound in the derivation. When using this rate, the weakening of the Mexican peso expected in case of Trump winning the elections corresponds to a downward movement. Trump, in turn, is the outcome analogue of Leave in the derivation. When comparing bookmakers, we have to make sure that the bookmaker we select for betting on Clinton accepts the Mexican peso as transaction currency. While all major bookmakers accept British pounds and most of them accept U.S. dollars, currencies like the peso are only accepted on a handful of websites. The upper part of Table 3 shows odds quoted by two major bookmakers on Nov. 6 and on Nov. 7, 2016 - one (two) day(s) before the elections. The lower half shows state prices and implied cuts calculated from these odds. The best available Trump odds on these days investable in Mexican pesos were offered by bet365, while the best available Clinton odds investable in U.S. dollars were offered by Betfair Sportsbook (the bookmaker, not the betting exchange). From Table 3 , we see that by

\begin{tabular}{lcccc} 
& \multicolumn{3}{c}{ Nov. 6 } & \multicolumn{2}{c}{ Nov. 7 } \\
\hline & bet365 & Betfair Sportsbook & bet365 & Betfair Sportsbook \\
\hline Clinton & $2 / 7$ & $2 / 7$ & $2 / 9$ & $1 / 5$ \\
Trump & 3 & $10 / 3$ & $7 / 2$ & $9 / 2$ \\
\hline Clinton & 0.778 & 0.778 & 0.818 & 0.833 \\
Trump & 0.25 & 0.231 & 0.222 & 0.182 \\
\hline Cut (in \%) & 2.78 & 0.85 & 4.04 & 1.52
\end{tabular}

Table 3: Top: Betting odds for the U.S. presidential elections 2016 from selected major bookmakers. Bottom: State prices and cuts calculated from these betting odds. Source: www.oddschecker.com, archived at www.webarchive.org on Nov. 6, 2016, 01:19, and on Nov. 7, 2016, 22:12.

betting on Clinton with bet365 and on Trump with Betfair Sportsbook, the cut is $0.85 \%$ on Nov. 6 and 0 on Nov. 7. On Nov. 6 , we start with 1 peso and put a stake of 0.778 pesos on Clinton with bet365. We convert the rest of 
0.222 pesos to dollars and bet $0.222 S_{\tau^{-}}^{\text {MXNUSD }}$ dollars on Trump with Betfair Sportsbook. This leads to a payoff of either 1 peso in case of Clinton winning, or to a payoff of $0.222 / 0.231 / d$ pesos after converting back from dollars for a Trump victory. In the absence of transaction costs, the strategy would have remained an arbitrage for any (correctly anticipated) conditional exchange rate return $d<0.222 / 0.231 \approx 0.96$, i.e., for a weakening of the Mexican peso by more than about $4 \%$ as a result of Trump winning. On Nov. 7 , we get from the same strategy either 1 peso or $0.182 / 0.182 / d$ pesos, requiring again only the correct anticipation of a weakening of the peso, regardless of its magnitude.

Transaction costs arising from the need to exchange currencies when implementing the strategies vary across market participants. For institutionals with access to interdealer FX platforms, Karnaukh, Ranaldo, and Söderlind 2015, Table 2) report effective average costs of $0.7 \mathrm{bp}$ for the GBPUSD pair. Increased competition from new service providers and among credit card companies has led to decreasing transactions costs for retail clients as well, even when considering all potential cost components. In total, they can be kept very small: First, internet betting providers regularly allow punters to pay by credit card without any fees. We explicitly checked that for bet365, where we would have placed our bet on Clinton in pesos. Second, many card issuers nowadays do not charge any foreign transaction fees, so that transaction costs can be easily limited to the markup of card network providers, i.e., the difference between their conversion rates and interbank mid-price. For major currency pairs such as the EURUSD rate, major networks like Visa or Mastercard have markups of 0.2-0.3\% above mid-market, and even for less liquid currencies like the Norwegian kroner, a US-based investor would face markups of only $0.3-0.6 \% 8$ Given that the Mexican peso is more liquid than the Norwegian kroner (BIS, 2016, p. 5), we take these values as an upper bound for the proportional transaction costs $\gamma$ (see Section 3.1) a punter

\footnotetext{
${ }^{8}$ See, e.g., https://nomadgate.com/visa-vs-mastercard-exchange-rate/.
} 
would have faced for the peso bet. Still assuming $d=0.9$, plugging $\gamma=0.3 \%$ into equation (24) on June 16, when the risk-neutral Remain probability was $61.9 \%$, yields a conservative estimate for transaction costs related to Strategy 1 in the Brexit case of $0.003(1-0.619+1 / 0.9)=0.0045$ or $0.45 \%$. For the U.S. presidential elections, using $\gamma=0.6 \%$, a risk-neutral Clinton probability of $77.8 \%$, and a cut of $c=0.85 \%$ on Nov. 6 gives transaction costs of about $0.006(1-0.222+(0.778-0.0085) / 0.9 / 0.778)=0.0112$ or $1.12 \%$. Hence, in addition to getting the direction of the exchange movements right, the movements would have had to be large enough to cover these transaction costs for our strategies to be profitable.

\subsection{Stochastic Conditional Returns}

In Section 4.1, we placed bets a few days before the events, and we assumed the conditional exchange rate reactions after the respective events to be deterministic and known ex ante. In this section, we will implement the strategies at an earlier point in time, i.e., one month before the events, and we will assume exchange rates to be lognormally distributed conditional on the possible event outcomes. Hanke et al. (2019) show that the mixture of lognormals model prices observed FX option prices prior to the events with high precision when using risk-neutral event outcome probabilities implied from observed betting odds. As a by-product of their analysis, they estimate parameters of the conditional return distributions. As an illustrative example, we use the parameter values they provide in their Table 2 for the first day when one-month FX options were traded that expired after the respective events.

In Section 4.1, we found that the best betting odds observed in both markets shortly before the respective events implied a cut of 0 . We find many such occasions in the weeks before the U.S. presidential elections as well: a cut of zero is observable on 7 out of 14 days for which we have data in the month before the elections. In contrast, this holds only for 2 out of 


\begin{tabular}{c|r|r|r}
\multicolumn{2}{c|}{ GBPUSD } & \multicolumn{2}{c}{ USDMXN } \\
\hline$\mu_{\mathrm{L}}(\tau-t)$ & -8.66 & $\mu_{\mathrm{T}}(\tau-t)$ & 7.49 \\
$\mu_{\mathrm{R}}(\tau-t)$ & 1.87 & $\mu_{\mathrm{N}}(\tau-t)$ & -1.38 \\
$\sigma_{\mathrm{L}} \sqrt{\tau-t}$ & 4.49 & $\sigma_{\mathrm{T}} \sqrt{\tau-t}$ & 6.35 \\
$\sigma_{\mathrm{R}} \sqrt{\tau-t}$ & 2.85 & $\sigma_{\mathrm{N}} \sqrt{\tau-t}$ & 5.35
\end{tabular}

Table 4: Parameters (in percent) of the conditional densities described in equations (12) and (13) as reported in Hanke et al. (2019, Table 2). Densities are estimated for the first day when one-month options were traded that expired after the respective events.

16 observations in the month before the Brexit referendum. On the days for which the cut is greater than zero, it is $1-1.5 \%$ for the U.S. presidential elections and 1.7-3.9\% for the Brexit referendum. When implementing the strategies discussed above on a day with a non-zero cut, the cut reduces the strategies' returns, which, in turn, reduces the resulting Sharpe ratios.

For the U.S. presidential elections, we observe betting odds that imply a cut of zero at time $t=$ Oct. 8, 2016 (one month before the elections). The interesting density to be exploited with our strategies is the conditional density in case of Trump winning the elections, which is indicated in Table 4 by the subscript T. For the exchange rate risk premium, we assume conservatively a value of $1 \%$ per month, which is way higher than usual estimates (see Section 3.2). Transaction costs to be included in the calculation of the Sharpe ratio can be computed using equation (24), similar to our calculations in Section 4.1 $]^{9}$ For a risk-neutral Clinton probability of $74.8 \%$ on Oct. 8, we estimate transaction costs of $0.006(1-0.748+1 / 0.9)=0.82 \%$. The resulting Sharpe ratio after accounting for conservative estimates for the risk premium and transactions costs would still be a very favorable $S R_{1} \approx(7.49-1-0.82) \sqrt{12} / 6.35=0.89 \sqrt{12}=3.09$ when ignoring the

\footnotetext{
${ }^{9}$ For a more exact estimation, $\bar{d}$ implied by the parameters in Table 4 plus the estimated risk premium could be used instead of $d$ in equation (24). This would yield slightly lower values for the transaction costs than for $d=0.9$, so our values are again conservative estimates.
} 
(negligibly low) one-month GBP interest rate. The fact that $\mu_{R}^{\mathbb{P}}$ is, conservatively estimated, 0.89 standard deviations away from 0 implies a probability of $N(0.89)=81.3 \%$ for a positive return of this strategy in case of Trump winning the elections, given our assumptions (in case of Clinton winning, the strategy always leads to a return of exactly 0 ).

For the GBPUSD density, the observed cut on May 25, 2016 (just less than one month before the referendum) was $2.8 \%$. For the risk-neutral exchange rate density in case of a majority voting for Leave, Table 4 provides a conditionally expected log return of $\mu_{L}^{\$}(\tau-t)=-8.66 \%$ and a standard deviation of $4.49 \%$. Transaction costs are estimated by using $\gamma=0.3 \%$ (see Section 4.1 in equation (24) and noting that the risk-neutral Remain probability on May 25 was 81.2\%: 0.003 $(1-0.188+(0.812-0.028) / 0.9 / 0.812))=0.0057$ or $0.57 \%$. Accounting for these transaction costs, for a conservative assumption of an exchange rate risk premium of $0.5 \%$ per month (see Section 3.2), and the cut of $2.8 \%$ when calculating the Sharpe ratio, we get $S R_{1} \approx(8.66-2.8-0.5-0.57) \sqrt{12} / 4.49=1.07 \sqrt{12}=3.70$ when ignoring the (very small) one-month USD interest rate. The fact that $\mu_{L}^{\mathbb{P}}$ is, conservatively estimated, 1.07 standard deviations away from 0 implies a probability of $N(1.07)=85.8 \%$ for a positive return of this strategy in case of an outcome of Leave, given our assumptions (in case of Remain, the strategy always leads to a return of exactly 0 ).

These numerical examples show that ignoring the numeraire-dependence of risk-neutral probabilities described in Section 2, i.e., offering the same odds for such events across currencies, may present a sizable risk to bookmakers and betting exchanges. Given the size of betting markets for these events mentioned in the introduction ( $£ 127 \mathrm{~m}$ and $£ 200 \mathrm{~m}$, respectively), this risk is not to be neglected: While the strategies described only result in arbitrage profits in a strict sense when assuming deterministic exchange rate reactions, relaxing this assumption results in highly favorable risk-return ratios, making the strategies "very good deals" or even approximate arbitrage opportunities 
in the sense of Shanken (1992), i.e., strategies with more than twice the market Sharpe ratio. For both events discussed here, there were several occasions when an effective cut of zero was "investable" in betting markets. In the absence of transaction costs (currency conversion), this means that to rule out losses, an investor just had to get the direction of the exchange rate reaction right, i.e., correctly forecast that a majority voting in favor of Leave (Trump) would result in a weakening of the pound (peso) relative to the dollar. Taking transaction costs into account, the required move of the exchange rate in the desired direction was still very small, i.e., around 0.5$1 \%$. For bets placed on days when the cut was positive, the exchange rate move would have been large enough to cover the cut on top of transaction costs. Implementing these strategies in practice is not completely risk-free, since nobody could have ruled out, e.g., a strengthening in the pound after a majority voting for Leave. However, the conditionally expected exchange rates implied in FX option prices before the respective events allowed both high forecast accuracy and high confidence in the direction of the conditional changes (see Hanke et al., 2019).

\section{Conclusion}

We have shown that for events whose outcomes affect exchange rates, risk-neutral event outcome probabilities are numeraire dependent. Simple no-arbitrage conditions have been derived and illustrated numerically under the assumption of deterministic exchange rate reactions after the respective events. For stochastic conditional exchange rate movements, the strategies described are no longer arbitrage opportunities, but may still be good deals, e.g., in terms of their Sharpe ratios. The conditions provided in this paper can and should be used by internationally active bookmakers to adjust their odds for such events according to the transaction currency. Even bookmakers who are only active domestically should adjust their odds accordingly to protect 
themselves from being exploited by the strategies described. Ignoring this effect, as seems to be current market practice, may lead to losses well in excess of a bookmaker's cut. This may present a high risk given the size of betting markets for such events. Our numerical examples have shown that both around the Brexit referendum and the U.S. presidential elections, odds available in betting markets would have allowed for very profitable strategies combining bets in different currencies. The effect described is not limited to betting markets, but occurs more generally for events risks that are correlated with exchange rates, and traded as financial contracts in different currencies.

\section{References}

BIS, 2016. Triennial central bank survey. Tech. rep., Bank for International Settlements, URL: http://www.bis.org/publ/rpfxf16fxt.pdf.

Björk, T., 2009. Arbitrage Theory in Continuous Time. Oxford University Press, third ed.

Cochrane, J., Saa-Requejo, J., 2002. Beyond arbitrage: Good-deal asset price bounds in incomplete markets. The Journal of Political Economy 108, 79119 .

Dixon, M., Pope, P., 2004. The value of statistical forecasts in the UK association football betting market. International Journal of Forecasting 20, 697-711.

Duffie, D., 2001. Dynamic Asset Pricing Theory. Princeton University Press, third ed.

Figlewski, S., 2010. Estimating the implied risk neutral density for the U.S. market portfolio. In: Bollerslev, T., Russel, J., Watson, M. (eds.), Volatility and Time Series Econometrics: Essays in Honor of Robert F. Engle, Oxford University Press. 
Franck, E., Verbeek, E., Nüesch, S., 2013. Inter-market arbitrage in betting. Economica 80, 300-325.

Golec, J., Tamarkin, M., 1991. The degree of inefficiency in the football betting market. Journal of Financial Economics 30, 311-323.

Hanke, M., Poulsen, R., Weissensteiner, A., 2019. Event-related exchange rate forecasts combining information from betting quotes and option prices. Journal of Financial and Quantitative Analysis forthcoming.

Hull, J., 2018. Options, Futures, and Other Derivatives. Pearson, 10th ed.

Jurek, J., Xu, Z., 2014. Option-implied currency risk premia, working paper, University of Pennsylvania and NBER.

Karnaukh, N., Ranaldo, A., Söderlind, P., 2015. Understanding FX liquidity. Review of Financial Studies 28, 3073-3108.

Lando, D., Nielsen, A., 2018. Quanto CDS spreads, working paper, Copenhagen Business School.

LeRoy, S., Werner, J., 2014. Principles of Financial Economics. Cambridge University Press, second ed.

Neuberger, A., 2016. How to beat the bookies with a brexit bet. The Conversation URL: https://theconversation.com/how-to-beat-the-bookies-witha-brexit-bet-60009.

Ritchey, R. J., 1990. Call option valuation for discrete normal mixtures. The Journal of Financial Research 13, 285-196.

Shanken, J., 1992. The current state of the arbitrage pricing theory. The Journal of Finance 47, 1569-1574. 
Vlastakis, N., Dotsis, G., Markellos, R., 2009. How efficient is the European football betting market? Evidence from arbitrage and trading strategies. Journal of Forecasting 28, 426-444.

Williams, L., Siegel, D. (eds.), 2013. The Economics of Gambling. Oxford University Press.

Wolfers, J., Zitzewitz, E., 2004. Prediction markets. Journal of Economic Perspectives 18, 107-126. 